\title{
An adaptive grid code for high resolution 1-D hydrodynamics of the solar and stellar transition region and corona
}

\author{
R. Betta ${ }^{1}$, G. Peres ${ }^{1}$, F. Reale ${ }^{1}$, and S. Serio ${ }^{1,2}$ \\ 1 Istituto e Osservatorio Astronomico di Palermo, Palazzo dei Normanni, I-90134 Palermo, Italy \\ 2 Istituto per le Applicazioni Interdisciplinari della Fisica/CNR, Via Archirafi 36, I-90123 Palermo, Italy
}

Received April 2; accepted July 31, 1996

\begin{abstract}
We present a new version of the PalermoHarvard hydrodynamic model of plasma confined in coronal loops, based on a regridding algorithm which ensures limited variations of thermodynamical variables across grid points. The code provides an accurate description of the dynamics of the chromosphere-corona transition region, in spite of the very steep temperature and density gradients, even during flare-like transients.

The new model can be used to synthesize, together with X-ray emission, the optically thin emission originating from the transition region, mainly in the EUV band. Here we show sample results.
\end{abstract}

Key words: methods numerical - hydrodynamics Sun: transition region - Sun: corona — Sun: flares Sun: UV radiation

\section{Introduction}

Hydrodynamical loop models have been traditionally used as a tool to understand the physics and the thermodynamics of coronal loops on the sun and the stars, in particular during complex and energetic phenomena such as flares. There have been many applications of hydrodynamical loop models to the study of confined solar plasma (Nagai 1980; Peres et al. 1982; Cheng et al. 1983; Doscheck et al. 1983; Cheng et al. 1984; Nagai \& Emslie 1984; Fisher et al. 1985a-c; MacNeice 1986; Mariska 1987). The PalermoHarvard code (henceforth, PH, Peres et al. 1982), in particular, has been extensively applied to many problems of coronal physics, including studies of loop stability, analysis of physical processes influencing the thermal flare (Pallavicini et al. 1983; Peres \& Reale 1993a,b; Reale \& Peres 1995), diagnostics of the location of the energy release in solar flares (Peres et al. 1987; Antonucci et al.

Send offprint requests to: betta@oapa.astropa.unipa.it
1987; Antonucci et al. 1993); the code has also been used to model a stellar flare observed by the Einstein satellite (Reale et al. 1988) allowing to infer the characteristic length of the flaring structure, to study the decay phase of solar (Serio et al. 1991; Jakimiec et al. 1992; Sylwester et al. 1993) and stellar flares (Reale et al. 1993), in order to devise diagnostics of flare conditions from the decay phase, and to study microflares (Peres et al. 1993).

The lack of resolution in the chromosphere-corona transition region is a problem shared by almost all existing time-dependent hydrodynamical loop models, including the $\mathrm{PH}$ code. In the transition region the temperature rises by two orders of magnitude to its typical coronal values $\left(\sim 10^{6} \mathrm{~K}\right)$, over a distance range shorter than $100 \mathrm{~km}$, which is a fraction $\sim 1 \%$ or less of typical loop lengths; the density undergoes a symmetrical decrease by two orders of magnitude. These very steep temperature and density gradients complicate the numerical resolution of the equations. Furthermore the transition region is strongly variable and dynamic: for instance it rapidly moves and steepens during flares and transient phenomena observable in the corona.

Since appropriate diagnostics of the very steep transition region requires the study of many optically thin spectral lines emitted mostly in the EUV band, which originate there, well sampled hydrodynamic solutions are indispensable for a correct interpretation of the data from space instruments such as those on board SOHO. As we will show, a good resolution in the transition region may also influence the solution in the corona; in fact, the evaluation of the conductive flux and mass motions in the transition region also determines the quality of the results in the rest of the atmosphere.

Here we present an updated version of the $\mathrm{PH}$ code, which describes the transition region with a resolution adequate to the requirements. To achieve this goal we have devised a regridding algorithm with non-uniform spacing which samples and accurately computes temperature, 
density and velocity everywhere in the loop system and maintains this resolution during the evolution by changing the grid with time so as to adapt it to the evolving solution. A uniform grid with high spatial resolution would not be appropriate, because of the inordinately high number of points and computer resources needed. A fixed grid with an unequal distribution of points would equally be inappropriate since the transition region rapidly moves and steepens during a typical flare evolution: after a few seconds of time integration an initially well distributed grid would no longer remain adequate.

Some authors have used a re-adaptive grid to compute the equations during the evolution of a flare but limited their analysis to the first few seconds, and in fact at best only to the rising phase of the event. In a few cases these limitations were necessary because a complete computation required an unaffordable amount of computer time (McClymont et al. 1983; Fisher et al. 1985; MacNeice 1986), in others the simulations were subject to immediate numerical instability (Gan et al. 1991).

In the previous version of the Palermo-Harvard numerical code (Peres et al. 1982), the hydrodynamical equations, translated into difference equations, are solved on a fixed spatial grid whose spacing changes along the spatial domain with a logarithmic law, finer in the chromosphere and coarser in the corona. Such a grid assures a number of points in the transition region larger than that obtainable with a uniform grid with an equal total number of points thus providing a partial solution to the need of spatial resolution. During the first phases of a flare, in fact, the steepened transition region typically moves towards the lower atmosphere where the spacing is finer. This choice of grid, however, is only partially satisfactory: temperature and density gradients are usually so steep during a flare that in practice, when using 256 points along a typical active region coronal loop of half-length $210^{9} \mathrm{~cm}$, only two or three grid points are in the transition region.

In order to satisfy these requirements we have devised an algorithm which generates a self-adaptive grid so as to maintain a fractional variation of temperature, density and pressure between adjacent grid points always below a chosen value. We will show how the implementation of this adaptive grid has significantly improved the results of the $\mathrm{PH}$ code. In Sect. 2 we describe the regridding algorithm, showing sample simulations in Sect. 3 and examining the synthesis of spectra in Sect. 4; in Sect. 5 we discuss our results and draw our conclusions.

\section{The numerical scheme}

The code solves the mass, momentum and energy conservation equations for a compressible fluid numerically using a finite difference scheme. The model is one-dimensional, since the plasma is confined in a semicircular loop where plasma motion and heat flow are allowed only along the magnetic field lines. Symmetry conditions allow us to consider only one half of the loop:

$$
\begin{aligned}
& \frac{\mathrm{d} n}{\mathrm{~d} t}=-n \frac{\partial v}{\partial s} \\
& n m_{\mathrm{H}} \frac{\mathrm{d} v}{\mathrm{~d} t}=-\frac{\partial p}{\partial s}+n m_{\mathrm{H}} g+\frac{\partial}{\partial s}\left(\mu \frac{\partial v}{\partial s}\right)
\end{aligned}
$$

$\frac{\mathrm{d} \mathcal{E}}{\mathrm{d} t}+w \frac{\partial v}{\partial s}=\mathcal{Q}-n^{2} \beta \mathcal{P}(T)+\mu\left(\frac{\partial v}{\partial s}\right)^{2}+\frac{\partial}{\partial s}\left(\kappa T^{5 / 2} \frac{\partial T}{\partial s}\right)$

$p=(1+\beta) n k_{\mathrm{B}} T$

$\mathcal{E}=\frac{3}{2} p+n \beta \chi$

$w=\frac{5}{2} p+n \beta \chi$

where $n$ is the hydrogen number density; $t$ is the time, $s$ is the field line coordinate; $v$ is the plasma velocity; $m_{\mathrm{H}}$ is the mass of hydrogen atom; $p$ is the pressure; $g$ is the component of gravity parallel to field line; $\mu$ is the viscosity; $\beta=n_{\mathrm{e}} / n$ is the ionization fraction where $n_{\mathrm{e}}$ is the electron density; $T$ is the temperature; $\kappa$ is the thermal conductivity ( $\simeq 910^{-7}$ erg $\left.\mathrm{cm}^{-1} \mathrm{~s}^{-1} \mathrm{~K}^{-7 / 2}\right) ; k_{\mathrm{B}}$ is the Boltzmann constant; $\chi$ is the hydrogen ionization potential; $\mathcal{P}(T)$ are the radiative losses per unit emission measure (Raymond et al. 1977); $\mathcal{Q}(s, t)$ is the volumetric power input to the solar atmosphere:

$\mathcal{Q}(s, t)=H_{\text {steady }}+H_{0} f(t) g(s)$;

$H_{\text {steady }}$ is the heating term, uniform in the corona and tabulated in the chromosphere, required to balance radiative and conductive losses for a static atmosphere; the second term is the impulsive heating triggering the flare.

The numerical scheme has been described previously by Peres et al. (1982) and Peres \& Serio (1984). The mass and momentum equations are solved with an explicit numerical scheme, with upwind differencing schemes used for the advective terms (Roache 1976). The energy equation is solved with an implicit scheme, which prevents numerical instabilities (Richtmyer \& Morton 1967; Fletcher 1988). Initial profiles of temperature and pressure are derived following the hydrostatic model of Serio et al. (1981), and matched with one of the chromospheric models of Vernazza et al. (1981).

As Dwyer (1983) pointed out, one of the key problems of numerical fluid dynamics is the simultaneous calculation of both the grid and the solution; in particular Dwyer suggests that the generation of the grid is perhaps more important than the approximation used for the spatial differencing.

The PH code has been designed on Eulerian coordinates. Lagrangian coordinates are appropriate to grant automatically a good resolution of the continuity equation 
but do not offer a significant advantage in the resolution of the fast moving steep transition region, one of the most critical aspects of the coronal loop dynamics and thermodynamics.

The use of a re-adaptive Eulerian grid generation method is probably the fittest choice to solve differential equations whose solutions present very steep gradients rapidly varying in time.

Another fundamental requirement of any finitedifference numerical grid scheme is the regularity of the spacing, i.e. the gradual change of the spacing along the grid (Thompson et al. 1985; Hawken et al. 1991; Tamamidis \& Assanis 1991). Lack of regularity, namely when the cell size varies abruptly along the grid, typically leads to numerical errors even worse than those caused by inadequate spatial grid resolution, as many authors have pointed out (Fisher et al. 1985a,b; Thompson et al. 1985; Gan et al. 1991; Hawken et al. 1991; Tamamidis \& Assanis 1991) and we ourselves have found.

In our approach we have essentially maintained the numerical scheme originally devised by Peres et al. (1982) for a time-independent spatial grid (with step changing according to a geometric progression of which the uniform grid is a special case). We have reorganized the code to account for the recurrent change of grid and succeeded in satisfying both the requirements of small relative change of the physical variables and of the cell sizes along the grid, with the twofold advantage of keeping both the number of points and the computing time low.

As a first fundamental condition the grid must satisfy the requirement that the fractional variation of temperature, density and pressure between two adjacent points remains below a fixed value $\alpha,{ }^{1}$

$\max \{\Delta T / T, \Delta n / n, \Delta p / p\} \leq \alpha$,

in the transition region and in the corona. Since this condition must be satisfied throughout the evolution of the solutions, the grid has to be modified according to Eq. (8), as discussed in the following, and the variables must be linearly interpolated on the new grid points whenever required.

Figure 1 shows a typical temperature profile vs. field coordinate (solid line), computed according to the static loop model of Serio et al. (1981), based on hydrostatic equilibrium and energy balance, as well as the irregularly distributed cell sizes that would be required to yield exactly a $10 \%$ jump of temperature between any two adjacent points (dots), and the cell sizes determined with our algorithm, discussed below in more details (dashed line). The loop shown is a typical active region one with halflength $210^{9} \mathrm{~cm}$, pressure at the base of the transition

$1 \quad$ We generally set $\alpha=0.1$ in our simulations thus allowing a maximum fractional variation of $10 \%$ of all the variables we are more interested in (i.e, temperature, density and pressure).

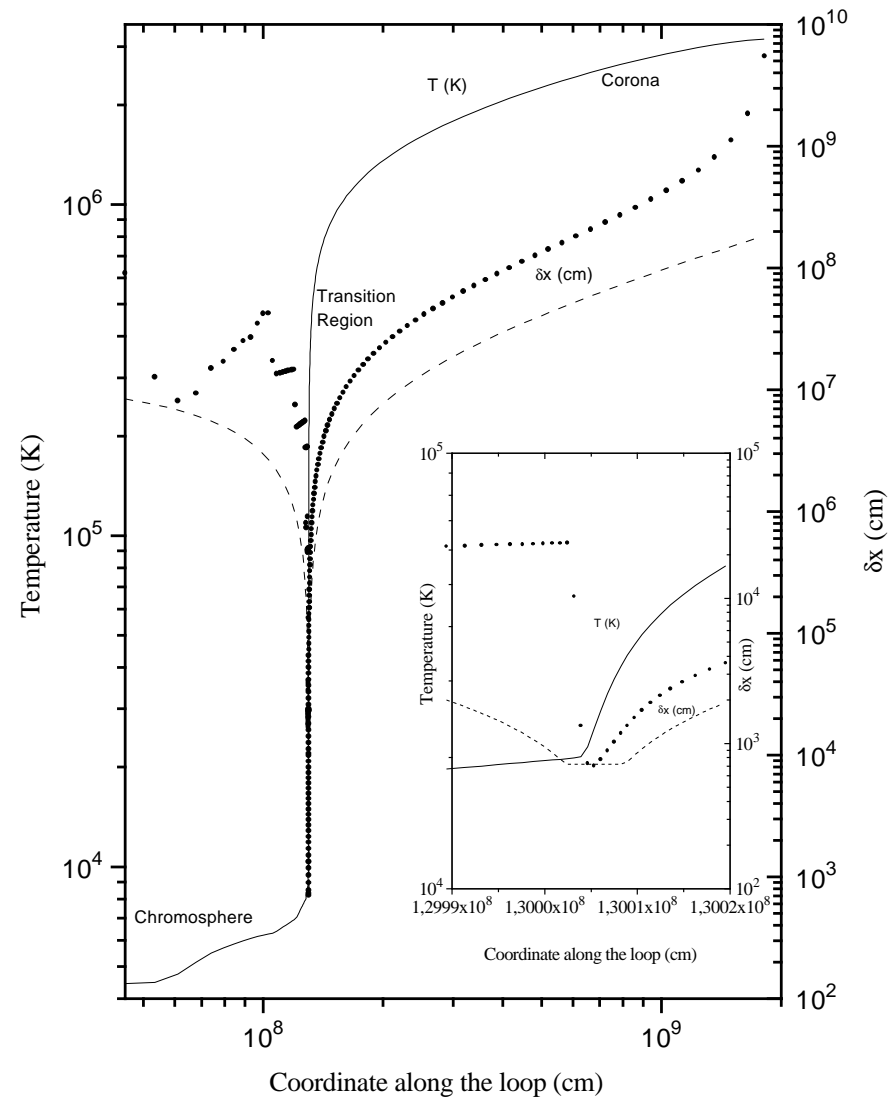

Fig. 1. Profiles of temperature vs. field line coordinates along one half of a symmetric loop of total length $410^{9} \mathrm{~cm}$ and base pressure 6 dyne $\mathrm{cm}^{-2}$ (solid line). The dots show the cell size required to resolve spatially the temperature with a fractional jump of exactly $10 \%$. The dashed line yields the cell size derived according to our algorithm. The inset shows an enlargement of the base of the transition region

region 6 dynes $\mathrm{cm}^{-2}$ and maximum plasma temperature at the top $3.210^{6} \mathrm{~K}$.

We have ascertained the importance of grid regularity with a simple test: if the initial hydrostatic atmosphere is used as initial condition for the numerical code, and $\mathcal{Q}(s, t)=H_{\text {steady }}$, we expect no evolution apart from some numerical noise; and in fact so was the case for the logarithmic grid fixed in time with the previous $\mathrm{PH}$ code (Peres et al. 1982), where the hydrostatic profiles remain essentially unchanged for integration times much longer than the characteristic dynamic and cooling times (i.e. longer than thousands of seconds). The non-uniform grid with spacing as in Fig. 1 (dots) instead yields significant changes in the profiles of temperature and density, because the grid is too irregular.

We have therefore made our grid more regular; in particular we have generated a grid finer in the transition region, where the highest resolution is needed, and whose spacing increases smoothly on both sides. The grid is split into three sections. In the first, the spacing 
decreases along field coordinates according to a geometric progression from the loop base to the transition region. The second is a small uniform grid (generally ten points), of high resolution placed just around the location where the smallest cell $\delta x_{0}$ is required, typically at the base of the transition region (see the inset of Fig. 1). Finally, in the third section, the spacing increases according to another geometric progression from the base of the transition region to the loop apex.

The whole grid is shown in Fig. 1 (dashed line). The two geometric progressions on the sides of the region with uniform spacing (in the first and in the third section) may have different ratios. The size of the $k$-th cell on either size of the uniform grid is determined as $\Delta x_{k}=\Delta x_{0} q^{k}$, $k=0,1,2,3, \ldots$. The code selects the values $q$ of the two ratios as the largest enforcing condition $(8)^{2}$. We dedicate particular care to the choice of the cells adjacent to the uniform grid, in order to make the size of the cells vary as gradually as possible; the first cell of each of the two geometric progressions $\Delta x_{0}$ satisfies the condition:

$\delta x_{0} \leq \Delta x_{0}<\delta x_{0} q$

and is determined so that the two progressions span exactly the remaining distance on each side of the loop. The initial temperature, pressure and velocity profiles are then linearly interpolated from the old grid onto the new grid.

After each step of time integration the algorithm checks the grid and verifies if the point where the smallest cell size is required by the condition (8) is still inside the section with uniform spacing, otherwise it generates a new grid with the same criterion described above. Since the value of the smallest size $\delta x_{0}$, its location along the loop, and the geometric ratio $q$ change frequently during the flare evolution, the number of points used along the loop is not constant but varies according to the requirements imposed by the conditions (8) and (9).

The small uniform grid at the base of the transition region has two advantages:

i) the computation of a new grid is not required at every time step; we therefore reduce the computing times and limit the errors inevitably introduced by interpolation.

ii) it gradually matches two sections with different spacing, reducing numerical errors significantly, as discussed above.

As for the influence of truncation errors in the new implementation, we focus on the energy Eq. (3) and, in particular, on the dominant thermal conduction term. The scheme has been originally devised to work on a geometric progression grid. In the section of the grid with uniform

2 However, the geometric ratio $q$ is always chosen in the interval $1<q<1.2$, to guarantee a fractional variation of the cell size below 20\% (Thompson et al. 1985; Fletcher 1988). spacing (the one with the steepest temperature gradient) the error is of the order of:

$\sim \frac{\kappa}{12}\left[\frac{\partial^{3}}{\partial x^{3}}\left(T^{5 / 2} \frac{\partial T}{\partial x}\right)\right] \Delta x^{2}$

In the non-uniform grid $\Delta x^{2}=\Delta x_{0}^{2} q^{2 k} \leq \Delta x_{0}^{2} 1.2^{2 k}$, apart from a factor of the order of unity. In the region of the small uniform grid, $\Delta x$ is very small, but, since the temperature derivatives are very big, the error is de facto dominated by the factor in parentheses. Far outside that region, $\Delta x$ is large but the temperature structure is much smoother, almost flat; since the critical part of our model is anyway the transition region, we take care that condition (8) is always satisfied.

The adoption of a new grid has significant consequences also on the value of the integration time step $\Delta t$. In particular, $\Delta t$ is selected as the minimum value among the following times evaluated for each $i$-th point in the grid:

- the Courant-Friedrichs-Lewy (CFL) time

$$
\tau_{\mathrm{CFL}_{i}}=\frac{\delta x_{i}}{c_{s_{i}}+\left|v_{i}\right|}
$$

where $\delta x_{i}$ is the distance between two grid points and $c_{s_{i}}$ is the sound speed

- the radiative time

$$
\tau_{\mathrm{rad}_{i}}=E_{i}\left(\frac{\partial E}{\partial t}\right)_{\operatorname{rad}_{i}}^{-1}=\frac{3 n_{i} k_{B} T_{i}}{\beta_{i} n_{i}^{2} P\left(T_{i}\right)}=\frac{3 k_{B} T_{i}}{\beta_{i} n_{i} P\left(T_{i}\right)}
$$

- the conductive time

$$
\tau_{\text {cond }_{i}}=\frac{E_{i}}{\left|\operatorname{div} F_{\operatorname{cond}_{i}}\right|}=\frac{21 n_{i} k_{B} T_{i}}{2 k\left|\frac{\partial^{2} T^{7 / 2}}{\partial x^{2}}\right|_{i}} .
$$

From (10) (see, for example, Richtmyer \& Morton 1967; Fletcher 1988) it is immediately evident that a reduction of $\delta x_{i}$ leads to a reduction of $\Delta t$. The conductive time is indeed $\propto \delta x_{i}^{2}$ but we have seen that during most of the flare simulations the time step is in fact governed by the CFL condition.

Occasionally the time step decreases below $10^{-5} \mathrm{~s}$, however the code continuously monitors $\Delta t$ and increases it whenever possible. Indeed if the time step remained below $10^{-5} \mathrm{~s}$, we would typically need $\sim 58$ hours of $\mathrm{CPU}$ time to simulate $600 \mathrm{~s}$ of a flare on a DEC Alpha 3000, since an integration time step takes $3.510^{-3} \mathrm{~s}$ (with 250 grid points along the loop). With this strategy computations take approximately one half this time. Our strategy is even more advantageous for runs covering the decay phase, where the time step can increase markedly.

We can also limit the time step imposing a lower size of the grid spacing $\delta x_{\text {min }}$, typically set at $100 \mathrm{~cm}$, with the minor disadvantage of a resolution at the base of the transition region sometimes slightly less than adequate for Eq. (8).

Following the suggestions by Thompson et al. (1985), we also make sure that the time step and, independently, 
the minimum space step $\delta x_{0}$ may change by, at most, a factor $q$. We have seen that such a condition helps in further reducing the numerical fluctuations sometimes present.

\section{Hydrodynamic simulations}

We have computed the time evolution of a static loop model with the new code. The loop is stable for very long times (several thousands of seconds): the initial configuration of the atmosphere does not change significantly when we let it relax maintaining only the steady heating. The solutions are still stable if we perturb the initial temperature and density profiles, for example giving small amplitude isobaric perturbations as those in Peres et al. (1982). Plasma velocities $(\leq 5 \mathrm{~km} / \mathrm{s})$ are always negligible compared to the local sound speed everywhere in the loop, and decrease in time in all the relaxation tests we have performed. We typically use relaxed atmospheres as initial configurations for any further investigation of the plasma evolution.

As a second representative test we report the results of a simulation of a flare in an active region loop and compare them to the results obtained with the previous $\mathrm{PH}$ code. We have chosen the model of the well-studied flare observed by SMM on the sun on November 121980 at 17:00 UT (Peres et al. 1987) obtained by using the previous PH code, with a satisfactory fitting of the XRP/SMM observations. The transient heating in Eq. (7) has been placed at the top of the loop with a Gaussian spatial distribution,

$g(s)=\exp \left[-\frac{\left(s-s_{0}\right)^{2}}{2 \sigma^{2}}\right]$

where $s_{0}=210^{9} \mathrm{~cm}, \sigma=510^{8} \mathrm{~cm}$; it is constant for the first 180 seconds and then decays exponentially with an e-folding time of 60 seconds; $H_{0}$ is $10 \mathrm{erg} \mathrm{cm}^{-3} \mathrm{~s}^{-1}$ while the steady heating term is uniform in the corona, with $H_{\text {steady }} \simeq 0.014 \mathrm{erg} \mathrm{cm}^{-3} \mathrm{~s}^{-1}$. The half-length of the loop is $L=210^{9} \mathrm{~cm}$, and the pressure at the base of the transition region is initially $p_{\mathrm{b}}=6$ dyne $\mathrm{cm}^{-2}$.

A first important point to check is how the more accurate numerical computation of temperature and density gradients in the transition region improves the results. It appears that we have overcome one of the difficulties which plagued the computation of hydrodynamics of flaring plasmas as discussed in the introduction.

Figure 2 shows the hydrodynamical results obtained with the new $\mathrm{PH}$ code along with the corresponding results from the previous code (dashed lines); the initial conditions are the same as in Fig. 1. We show results focusing on the transition region, because this is the part of the loop atmosphere where the new code is apt to probe the behavior of the plasma in a way inaccessible to the previous $\mathrm{PH}$ code. The parameters of the simulations are identical apart from the numerical grid. The time profiles of temperature and density sample the first 30 seconds
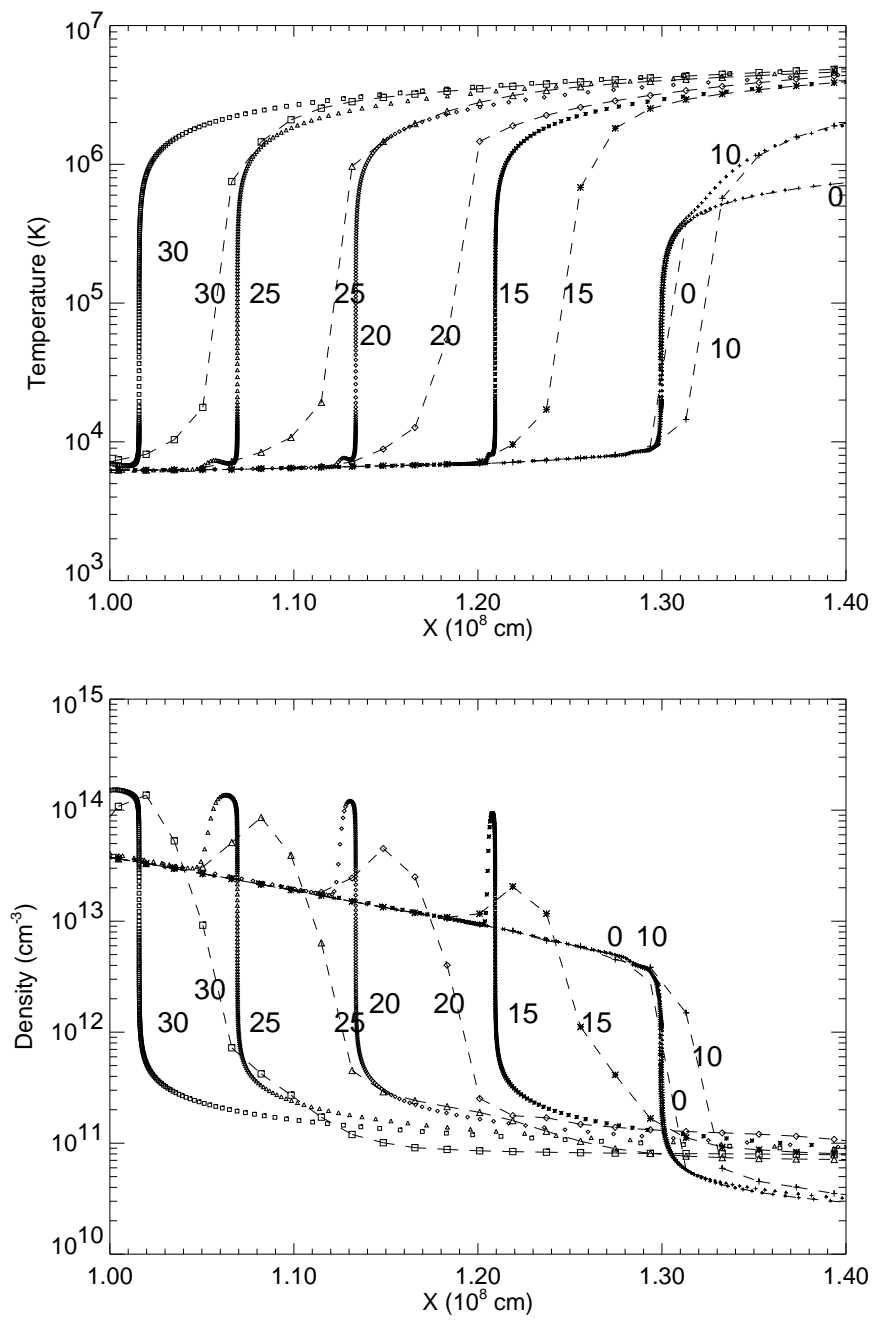

Fig. 2. Comparison between the evolution of the plasma temperature and density during a typical flare, computed with the new code, and that obtained with the previous code. The parameters of the simulation are reported in the text; the initial configuration of the atmosphere (time $=0 \mathrm{~s}$ ) is the one shown in Fig. 1. We have joined by short-dashed lines the points calculated with the previous code to guide the eye. The curves are taken every 5 seconds for a total time of 30 seconds. The transition region, both in the temperature and density profiles, moves from right toward the left after the beginning of the impulsive heating

of the flare evolution. The total number of grid points is also approximatively the same: the simulations of Peres et al. (1987) were done on a fixed grid with 256 logarithmically spaced points; now the number of points is typically around 275, and in this case varies from a minimum of 242 (at $10 \mathrm{~s}$ ) to a maximum of 343 (at $5 \mathrm{~s}$ ) along the whole loop.

Snapshots taken at the same times are different, and now with a significantly higher resolution, the computed gradients are also steeper. The plasma condensation, which typically forms in the chromosphere after a few 


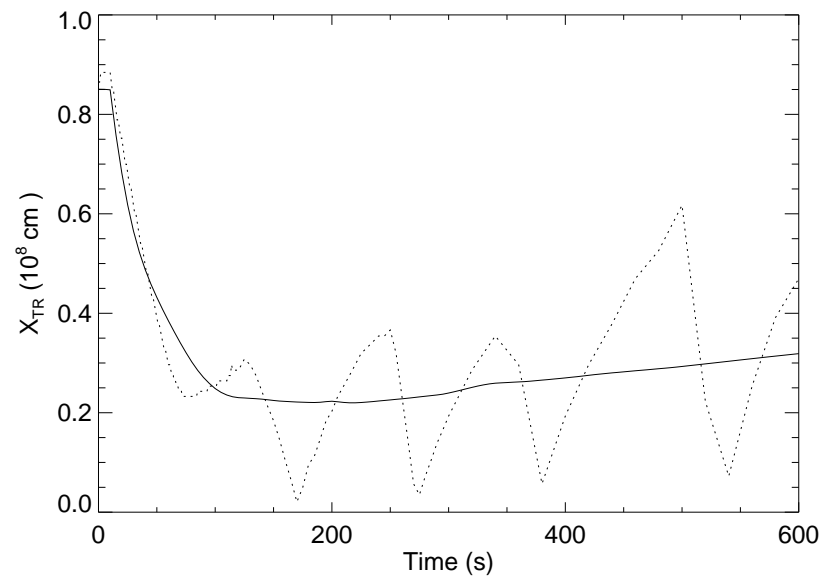

Fig. 3. Displacement of the transition region during the flare evolution. We have plotted vs. time the spatial coordinate of the point at $10^{5} \mathrm{~K}$. The results obtained with the new code (solid line) are compared with the previous ones (dashed line)

seconds since the impulsive heating is turned on $(\sim 15 \mathrm{~s}$ for this flare), is now resolved much better and the density maximum is higher. The transition region of the flaring atmosphere, at any time after $10 \mathrm{~s}$, is located lower than predicted with the previous code. These results show, once again, the improvement produced by the adaptive grid, since under-resolving the transition region results in an underestimation of the speed with which chromospheric evaporation occurs. The proper resolution therefore produces significantly more accurate results for the diagnostics of UV lines formed in the transition region and for the plasma dynamics during the chromospheric evaporation.

Another large difference with the old results is that we no longer find oscillations of the position of the transition region. This aspect is shown in Fig. 3 where the displacement along the loop of the volume element corresponding to a temperature $10^{5} \mathrm{~K}$ is plotted, both for runs using the old and new version of the code.

\section{Synthesis of the observables}

The most relevant innovation of the new version of the code is that we can now reliably synthesize the emission from the transition region, mostly in the EUV band, as well as that from the corona. The high resolution of the temperature structure in the transition region now allows a proper sampling in the temperature range of many EUV lines of the transition region.

We derive the predicted emission from temperature and density profiles obtained along the loop at different times during the flare evolution. The flux in a particular line, at a distance $R$ from the sun $(R \sim 1 \mathrm{AU})$, is calculated as:

$F \propto \frac{1}{4 \pi R^{2}} \int_{\Delta V} n_{\mathrm{e}}^{2} G(T) \mathrm{d} V$ where $n_{\mathrm{e}}=$ is the electronic density, $T$ is the plasma temperature, $V$ is the plasma volume, and $G(T)$ is the emission function which has been tabulated for most observed lines by several authors. We use Landini \& Monsignori Fossi (1990) tables as $G(T)$.

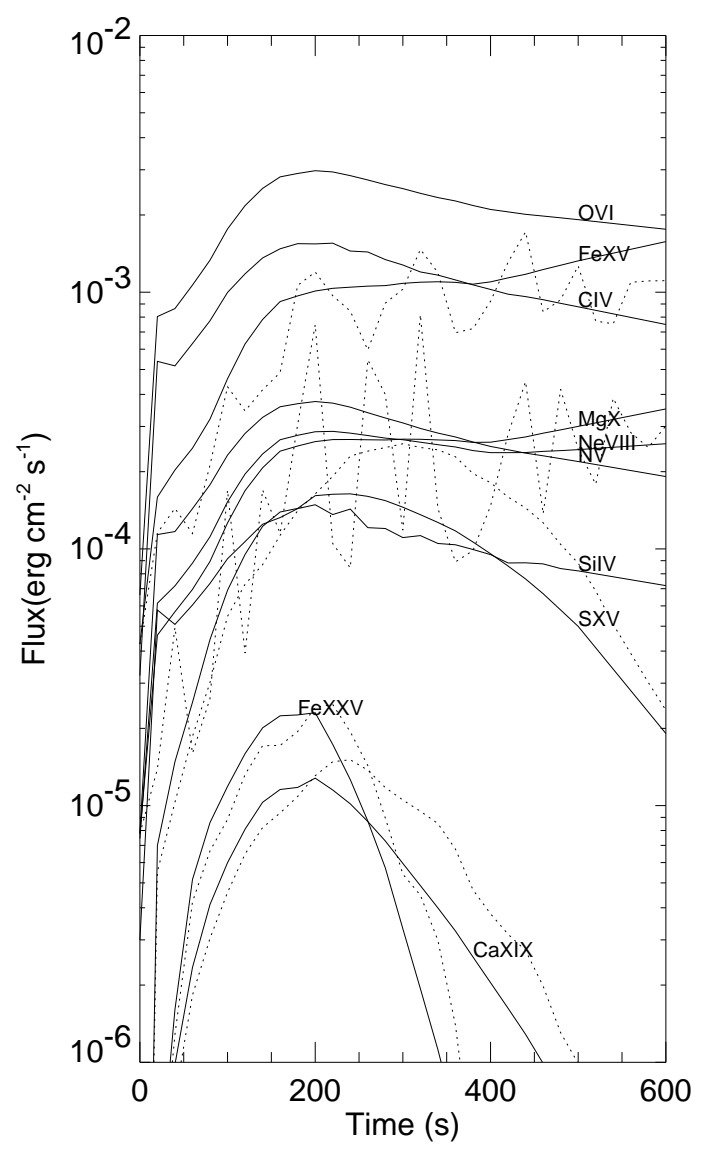

Fig. 4. Same flare as in Fig. 2: predicted light curves of the flare in ten important EUV and X-ray spectral lines which sample different temperatures of the transition region and corona synthesized with the results of the new code (solid lines). We also show for comparison, five of the light curves (Fe XXV, Ca XIX, S XV, Fe XV, Mg X) obtained with the previous PH code (dashed lines); the others were by far too noisy to be reported

As a test we have synthesized 10 spectral lines which are frequently observable by many satellites such as SMM (XRP), Yohkoh (BCS), and SOHO (EIT, CDS, SUMER). They form in a wide range of temperatures, spanning from the upper chromosphere to the high corona. They are: $\mathrm{Fe}$ XXV $\left(1.85 \AA\right.$, $\left.710^{7} \mathrm{~K}\right)$, Ca XIX $\left(3.17 \AA\right.$, $\left.310^{7} \mathrm{~K}\right)$, S $\mathrm{XV}\left(5.04 \AA, 1.610^{7} \mathrm{~K}\right)$, Fe XV $\left(284.15 \AA, 2.510^{6} \mathrm{~K}\right)$, $\mathrm{Mg} X\left(609.80 \AA, 10^{6} \mathrm{~K}\right)$, Ne VIII $\left(780.32 \AA, 6.310^{5} \mathrm{~K}\right)$, O VI $\left(1031.90 \AA, 410^{5} \mathrm{~K}\right)$, N V $\left(1238.80 \AA, 1.610^{5} \mathrm{~K}\right)$, C IV $\left(1548.20 \AA, 10^{5} \mathrm{~K}\right)$, Si IV (1402.77 $\left.\AA, 6.310^{4} \mathrm{~K}\right)$. We show the light curves in these lines in Fig. 4. The inadequate resolution of the previous $\mathrm{PH}$ code has not 
crucially affected the predictions for the X-ray spectral emission which originate at the highest temperatures, such as the Fe XXV, Ca XIX and S XV lines. These predicted curves successfully fitted the observed light curves of the November 12 flare.

Differences due to the numerical noise become more evident in the light curves of spectral lines, such as $\mathrm{Fe}$ $\mathrm{XV}$ and $\mathrm{Mg} \mathrm{X}$, which form at the lowest coronal temperatures, next to the transition region (see Fig. 4). As for the emission of the plasma at temperature below $10^{6} \mathrm{~K}$ (principally UV), we can synthesize it only by using the new version of the Palermo-Harvard code and therefore we have no other results to compare with.

\section{Discussion and conclusions}

The new version of the $\mathrm{PH}$ code we have presented, achieves an adequately high spatial resolution all along the loop from the corona to the chromosphere.

Beyond the obvious advantage of an accurate calculation of the plasma evolution even during very fast and dynamic phases such as the beginning of a flare, we now have the possibility to diagnose and/or predict plasma conditions in the transition region, and reliably derive line or band emission even in the EUV band. In this respect the resolution of the transition region, ensuring a fractional jump of temperature and density below $10 \%$, also implies a high sampling of the ranges of temperatures of practically all the transition region lines of interest, an important factor for accurate line synthesis.

The potential for new diagnostics now unfolds, therefore, and it is possible to compare model predictions with EUV observations directly. This is particularly promising in view of the upcoming data from the CDS, SUMER, EIT instruments on board the SOHO mission. As for coronal data, and more specifically, X-ray observations, comparison with the results of the previous version are comforting: while certainly some details of the evolution appear different, the global features and plasma characteristics remain qualitatively unaltered. The results developed with this new version of the code show that previous applications of the $\mathrm{PH}$ code, being limited in scope to the dynamics of the plasma in the corona were essentially correct.

The previous $\mathrm{PH}$ code has been extensively used to simulate and study stellar flares; a fortiori the new version can be applied to stellar flares as well. Solar X-ray flares are typically observed with fast flares modes and/or hard filters or lines, while stellar flares are observed with the same wide band instruments used for standard observations. The implication is that ROSAT/PSPC and EINSTEIN/IPC observations of stellar flares cover down to $0.1 \mathrm{keV}$ and $0.16 \mathrm{keV}$ respectively, and are sensitive to plasma at a few million degrees, i.e. in the transition region of the flare. The high resolution of the new code allows us to synthesize more accurately the emission in all of the instrumental band.

In conclusion this improved version of the $\mathrm{PH}$ code allows us to obtain reliable diagnostics in a very wide spectral band, and appears to be adequate to model observational data taken with new generation instruments, as we plan to do in the near future.

Acknowledgements. We acknowledge partial support from Ministero dell'Università e della Ricerca Scientifica e Tecnologica, and Consiglio Nazionale delle Ricerche.

\section{References}

Antonucci E., Dodero M.A., Peres G., et al., 1987, ApJ 322, 522

Antonucci E., Dodero M.A., Martin R., et al., 1993, ApJ 413, 786

Cheng C.C., Karpen J.T., Doscheck G.A., 1984, ApJ 286, 787

Cheng C.C., Oran E.S., Doscheck G.A., et al., 1983, ApJ 265, 1090

Doscheck G.A., Cheng C.C., Oran E.S., et al., 1983, ApJ 265, 1103

Dwyer H.A., 1983, "A discussion of some Criteria of Adaptive Gridding", in Adaptive Computational Methods For Partial Differential Equations, Babuska I., Chandra J., Flaherty J.E. (eds.) SIAM

Fisher G.H., Canfield R.C., McClymont A.N., 1985a, ApJ 289, 414

Fisher G.H., Canfield R.C., McClymont A.N., 1985b, ApJ 289, 425

Fisher G.H., Canfield R.C., McClymont A.N., 1985c, ApJ 289, 434

Fletcher C.A.J., 1988, Computational Techniques for Fluid Dynamics. Springer-Verlag, Berlin, Heidelberg

Gan W.Q., Fang C., Zhang H.Q., 1991, A\&A 241, 618

Hawken D.F., Gottlieb J.J., Hansen J.S., 1991, J. Comput. Phys. 95, 254

Jakimiec J., Sylwester B., Sylwester J., et al., 1992, A\&A 253, 269

Landini M., Monsignori Fossi B.C., 1990, A\&AS 82, 229

MacNeice P., 1986, Solar Phys. 103, 47

Mariska J.T., 1987, ApJ 319, 465

Nagai F., 1980, Solar Phys. 68, 351

Nagai F., Emslie A.G., 1984, ApJ 279, 896

Pallavicini R., Peres G., Serio S., et al., 1983, ApJ 270, 270

Peres G., Reale F., 1993a, A\&A 267, 566

Peres G., Reale F., 1993b, A\&A 275, L13

Peres G., Reale F., Serio S., Pallavicini R., 1987, ApJ 312, 895

Peres G., Reale F., Serio S., 1993, Proceedings of G.S.Vaiana Memorial Symposium, Palermo, 22-26 June 1992. In: Linsky J.F. and Serio S. (eds.)

Peres G., Rosner R., Serio S., Vaiana G.S., 1982, ApJ 252, 791

Peres G., Serio S., 1984, Mem. S. A. It. 55, 4

Raymond J.C., Smith B.W., 1977, ApJS 35, 419

Reale F., Peres G., 1995, A\&A 299, 225 
Reale F., Peres G., Serio S., et al., 1988, ApJ 328, 256

Reale F., Serio S., Peres. G., 1993, A\&A 272, 486

Richtmyer R.D., Morton K.W., 1967, Difference methods for initial value problems, Second edition. Interscience Publishers a division of John Wiley

Roache P., 1976, Computational Fluid Dynamics. Hermosa Publishers

Serio S., Peres G., Vaiana G.S., et al., 1981, ApJ 243, 288
Serio S., Reale F., Jakimiec J., et al., 1991, A\&A 241, 197 Sylwester B., Sylwester J., Serio S., et al., 1993, A\&A 267, 586 Tamamidis P., Assanis D.N., 1991, J. Comput. Phys. 94, 437

Thompson J.F., Warsi Z.U.A., Mastin C.W., 1985, Numerical Grid Generation, North-Holland, New-York, Amsterdam, Oxford

Vernazza J.E., Avrett E.H., Loeser R., 1981, ApJS 45, 635 